\title{
Cannabinoids for spasticity due to multiple sclerosis or paraplegia: A systematic review and meta-analysis of randomized clinical trials
}

\author{
Victoria P. da Rovare ${ }^{a}$, Gabriel P.A. Magalhães ${ }^{a}$, Guilherme D.A. Jardini ${ }^{a}$, Matheus L. Beraldo ${ }^{a}$, \\ Mariel O. Gameiro ${ }^{\mathrm{a}}$, Arnav Agarwal ${ }^{\mathrm{b}, \mathrm{c}}$, Gustavo José Luvizutto ${ }^{\mathrm{d}}$, Lucas Paula-Ramos ${ }^{\mathrm{e}, *}$, \\ Samira Esteves Afonso Camargo ${ }^{\mathrm{e}}$, Luciane Dias de Oliveira ${ }^{\mathrm{e}}$, Rodrigo Bazan ${ }^{\mathrm{d}}$, Regina El Dib ${ }^{\mathrm{e}, \mathrm{f}, \mathrm{g}}$ \\ a São Paulo State University (Unesp), Botucatu Medical School, São Paulo, Brazil \\ b Department of Clinical Epidemiology \& Biostatistics, McMaster University, Hamilton, Ontario, Canada \\ ${ }^{\mathrm{c}}$ Faculty of Medicine, University of Toronto, Toronto, Ontario, Canada \\ d São Paulo State University (Unesp), Department of Neurology, Botucatu Medical School, Brazil \\ e São Paulo State University (Unesp), Department of Biosciences and Oral Diagnosis, Institute of Science and Technology, São Paulo, Brazil \\ ${ }^{\mathrm{f}}$ São Paulo State University (Unesp), Department of Anesthesiology, São Paulo, Brazil \\ g McMaster Institute of Urology, McMaster University, St. Joseph's Healthcare, Hamilton, Canada
}

\section{A R T I C L E I N F O}

\section{Key-words:}

Cannabinoids

Spasticity

Multiple sclerosis

Paraplegia

\begin{abstract}
A B S T R A C T
Objectives: Spasticity remains highly prevalent in patients with spinal cord injury and multiple sclerosis. To summarize the effects of cannabinoids compared with usual care, placebo for spasticity due to multiple sclerosis (MS) or paraplegia.

Methods: Searches of MEDLINE, EMBASE, CENTRAL and LILACS to March 2017 were performed to identify randomized controlled trials. The primary outcomes were spasticity and spasm frequency. The criteria were any patient with MS and spasticity affecting upper or lower limbs or both, and that had a confirmed diagnosis of MS based on validated criteria, or however defined by the authors of the included studies.

Results: 16 trials including 2597 patients were eligible. Moderate-certainty evidence suggested a non-statistically significant decrease in spasticity (standardized mean difference (SMD) 0.36 [confidential interval (CI) $95 \%-0.17$ to $0.88 ; \mathrm{p}=0.18$; I2 = 88\%]), and spasm frequency (SMD 0.04 [CI 95\% -0.15 to 0.22$]$ ). There was an increase in adverse events such as dizziness (risk ratio (RR) 3.45 [CI 95\% 2.71-4.4; p = 0.20; I2 = 23\%]), somnolence (RR 2.9 [CI 95\% 1.98-4.23; p = 0.77; I2 = 0\%]), and nausea (RR 2.25 [CI 95\% 1.62-3.13; p = 0.83; I2=0\%]).

Conclusions: There is moderate certainty evidence regarding the impact of cannabinoids in spasticity (average 0.36 more spasticity; 0.17 fewer to 0.88 more) due to multiple sclerosis or paraplegia, and in adverse events such as dizziness (419 more dizziness/1000 over 19 weeks), somnolence (127 more somnolence/1000 over 19 weeks), and nausea (125 more somnolence/1000 over 19 weeks).
\end{abstract}

\section{Introduction}

Spasticity can be considered disabling when it involves severe functional problems, and the management is essential to prevent further deterioration in function. If not managed in a timely manner, spasticity can lead to diminished activity, and problems with daily living activities (ADL) such as gait, feeding, washing, dressing and toileting. ${ }^{1}$ Over time, spasticity may cause muscle pain, stiffness or spasms, trouble moving, impaired ability to stand and walk, difficulty eating and speaking, contracture leading to joint and bony deformity and even incontinence episodes ${ }^{2}$

Spasticity remains highly prevalent in patients with spinal cord injury (SCI) and multiple sclerosis (MS). In SCI patients, the lesion of the neurons in the spinal cord results in upper motor neuron syndrome with a prevalence of $65 \%$ to $78 \%$ in the first year post-injury, ${ }^{3}$ and in MS, the same is caused by the demyelination of nerve fibers of spinal cord and is present in $84 \%$ of North American cases. ${ }^{4}$

The treatment of multiple sclerosis has changed over the last years.

\footnotetext{
* Corresponding author at: Regina El Dib, Department of Biosciences and Oral Diagnosis, São Paulo State University (Unesp), Institute of Science and Technology, São José dos Campos, Francisco José Longo 777, São Dimas, São José dos Campos, SP, 12245-000, Brazil.

E-mail addresses: victoriarovare@gmail.com (V.P. da Rovare), beelz.magalhaes@gmail.com (G.P.A. Magalhães), guilhermejardini@gmail.com (G.D.A. Jardini), beraldoa@hotmail.com (M.L. Beraldo), marielorsi@gmail.com (M.O. Gameiro), arnav.mcmaster@gmail.com (A. Agarwal), gustavo.luvizutto@uftm.edu.br (G.J. Luvizutto), lucas93paula@hotmail.com.br (L. Paula-Ramos), samira@ict.unesp.br (S.E.A. Camargo), luciane@ict.unesp.br (L.D. de Oliveira), bazan.r@terra.com.br (R. Bazan), eldib@fmb.unesp.br (R. El Dib).
} 
The existing options for the treatment of spasticity, such as baclofen, tizanidine, benzodiazepines, morphine, and botulinum toxin present great limitations requiring frequent administration of high doses, often causing incapacitating side effects, and having a large number of patients who are unresponsive to therapy. ${ }^{5}$ Overall, the treatment of MS comprises three main groups: i) treatment of the acute attack; ii) prevention of future attacks by reducing triggers and use of disease-modifying therapies; and iii) symptomatic treatments of neurological difficulties such as spasticity, pain, fatigue, and bladder dysfunction. Thus, there is an urge for new treatment approaches, represented in the last decade by a number of publications regarding the use of cannabinoids and their effect in the endocannabinoid system.

The endogenous cannabinoids anandamide, 2-arachidonyl glycerol (2-AG) acts on specific cannabinoid receptors: CB1 receptors, present mostly in the CNS; and CB2 receptors, located in the CNS and extensively in the periphery (specially the immune system). ${ }^{6}$ Cannabis sativa $\mathrm{L}$. contains 60 or more cannabinoids, the most abundant of which are delta9-tetrahydrocannabinol (THC) and cannabidiol (CBD). ${ }^{6}$ THC is a partial $\mathrm{CB} 1$ receptor agonist providing analgesia, muscle relaxation, anti-emesis, appetite stimulation and psychoactivity. ${ }^{6}$ CBD has anticonvulsant, muscle relaxant, anxiolytic, neuroprotective, antioxidant and antipsychotic activity and has been shown to reduce the anxiogenic and psychoactive effects of THC. ${ }^{6}$ Both endogenous and exogenous cannabinoids have been shown to have an anti-spasticity effect in the recognized animal model of MS spasticity, and treatments that include THC and CBD have great potential for treating spasticity both in MS and SCI. ${ }^{6}$

There are a variety of new medications yet to be approved by governments that explore the effects of cannabinoids in the treatment of cancer pain, neuropathic pain, epilepsy, metabolic syndrome, inflammation, psychiatric disorders, spasticity in multiple sclerosis and spinal cord injury and other conditions, not to mention the possibility of using in-natura plant extracts. ${ }^{7}$

A recent systematic review of 79 trials addressing patient-important outcomes and including over 6000 patients reported that cannabinoids was associated with a reduction in spasticity as well as with improvements in nausea and vomiting due to chemotherapy, and weight gain in HIV infection, sleep disorders, and Tourette syndrome. ${ }^{8}$ A more specific metaanalysis on chronic pain and psychiatric problems concluded that there is high-quality evidence supporting the use of marijuana or cannabinoids. ${ }^{9}$

We therefore undertook a systematic review of all randomized controlled trials (RCTs) comparing any type of cannabis extract or cannabinoid-based medication with usual care or placebo focusing on patient-important outcomes for multiple sclerosis and spinal cord injury patients with spasticity. The aim of this systematic review and metanalysis is to look into more detail on the use of cannabinoids for these particular conditions. The intent to highlight specifically spasticity is due to the recent regulation of 1:1 THC:CBD oromucosal spray as a prescription medication in Brazil for patients with multiple sclerosis resistant to the current existing treatment.

\section{Methods}

The Cochrane Handbook for Intervention Reviews. ${ }^{10}$ guided our choice of methods. This review adheres to the Preferred Reporting Items for Systematic Reviews and Meta-analyses (PRISMA) Statement ${ }^{11}$ and, the Quality of Reporting of Meta-analyses QUOROM ${ }^{12}$

\subsection{Eligibility criteria}

- Study design: RCTs.

- Participants: patients with spasticity due to MS or paraplegia (i.e. complications of paralysis of the legs and lower parts of the body) affecting upper or lower limbs or both, and that had a confirmed diagnosis of MS based on validated criteria, or however defined by the authors of the included studies, and regardless the subgroup of the disease such as relapsing remitting, primary progressive and secondary progressive MS.

- Interventions: cannabis plant, with any compounds such as delta-9tetrahydrocannabinol (THC) and/or cannabidiol (CBD), regardless the type of extracts (e.g. oil, hash, tinctures).

- Comparators: usual care, placebo or no intervention.

- Patient-important outcomes: the primary outcomes were spasticity, and spasm frequency and severity. Secondary outcomes were pain measured by any validated scale, bladder function; cognitive function; ADLs; and occurrence of any adverse events (dizziness, somnolence, nausea, dry mouth).

Eligible studies followed patients for a minimum of two weeks. We did not consider studies reported as conference abstracts due to the lack of complete information they contained.

\subsection{Data source and searches}

A previous review. ${ }^{8}$ with similar inclusion criteria identified studies using cannabinoid treatment for different outcomes up to April 2015. We selected from the previous review ${ }^{8}$ only the RCTs that analyses the use of cannabis-based medication for spasticity, and developed a search strategy (Appendix Figure A1) for MEDLINE, EMBASE, Cochrane Controlled Trials Register (CENTRAL) and LILACS up to March 20, 2017. The review authors scrutinised the reference lists of the identified relevant studies for additional citations. We consulted clinical specialists and contacted authors of included trials where appropriate to obtain unpublished data.

\subsection{Selection of studies}

After identifying all potentially eligible studies by the literature search and obtaining all of their full-text articles, teams of two reviewers independently evaluated these studies for eligibility. Disagreements were resolved through discussion with third party adjudication. We calculated the agreement, using kappa statistic, between reviewers for full-text screening.

\subsection{Data extraction and risk of bias assessment}

The following data were extracted independently by three pairs of reviewers using a pre-standardized form that included characteristics of the study design, participants, interventions, outcomes event rates and follow-up. Authors of the eligible studies were contacted by reviewers to identify missing data and confirm data accuracy. As there was multiple publication of the same study, we decided to quote all these references under results section.

The pairs of reviewers assessed risk of bias separately by using a modified version of the Cochrane Collaboration's tool ${ }^{13}$ which includes nine domains: adequacy of sequence generation, allocation sequence concealment, blinding of participants and caregivers, blinding of data collectors, blinding for outcome assessment, blinding of data analysts, incomplete outcome data, selective outcome reporting, and the presence of other potential sources of bias not accounted for in the previously cited domains ${ }^{14}$ A low risk of bias was designated for incomplete outcome data, loss to follow-up of less than $10 \%$ and a difference of less than $5 \%$ in missing data in intervention and control groups. If needed, reviewers discussed with a third party adjudication to resolve disagreements.

\subsection{Certainty of evidence}

Reviewers used the Grading of Recommendations Assessment, Development and Evaluation (GRADE) methodology for certainty of evidence. Each outcome was rated high, moderate, low, or very low. ${ }^{14}$ Detailed GRADE guidance was used to evaluate overall risk of bias, ${ }^{15}$ imprecision, ${ }^{16}$ inconsistency, ${ }^{17}$ indirectness, ${ }^{18}$ and publication bias ${ }^{19}$ and results were summarized in an evidence profile. 


\subsection{Data synthesis and statistical analysis}

We calculated pooled risk ratios (RRs) for dichotomous outcomes and standardized mean differences (SMD) for continuous variables with the associated 95\% CIs using random-effects models with the MantelHaenszel statistical method. Absolute effects and 95\% CI were calculated by multiplying pooled RRs and $95 \%$ CI by baseline risk estimates derived from the largest of included RCTs in the meta-analysis. For dealing with missing data, we used complete case as our primary analysis; that is, we excluded participants with missing data. If results of the primary analysis achieved statistical significance, we planned to conduct sensitivity analyses to test the robustness of those results; however, we were not able to because the primary outcomes did not reach a statistical significance.

Results were assessed by each study using different scales. Variability in results across studies was undertaken by using $\mathrm{I}^{2}$ statistic and the $\mathrm{P}$ value obtained from the Cochrane chi square test. Our primary analyses were based on eligible patients who had reported outcomes for each study (complete case analysis).

We focused on publication bias through visual inspection of funnel plots for outcomes addressed in 10 or more studies. We used Review Manager (RevMan) (version 5.3; Nordic Cochrane Centre, Cochrane) for all analyses. ${ }^{20}$

\section{Results}

\subsection{Selection of titles}

Our search strategy focusing on publications since the last review identified 124 potential citations (Fig. 1). After title and abstract screening, we assessed the full-text articles of 33 relevant citations. In addition, we identified seven potentially eligible publications in our search strategy, and one further study through contact with an expert in the field. We subsequently assessed the eligibility of 41 full-text articles and excluded 17 studies (Fig. 1). Twenty-four publications documenting 16 RCTs involving 2597 participants seven cross-over, ${ }^{21-30}$ and nine parallel group ${ }^{31-44}$ proved eligible. The inter-observer agreement for the full-text screening was rated as good (kappa 0.65).

\subsection{Study characteristics}

Table 1 describes study characteristics related to study design, country, number of participants, mean age, eligibility criteria, and follow-up. Thirteen studies ${ }^{24,28-39,24,40,44}$ were conducted in Europe, one in the USA ${ }^{22,26}$ one in Canada, ${ }^{23,25}$ and one did not report the country where the RCT was conducted. ${ }^{27}$ Randomized trial sample sizes ranged from $11^{23,25}$ to $630^{41-43}$. Participants were typically aged between 40 and 60 years, and were followed for two to 19 weeks. Inclusion criteria usually included having multiple sclerosis, ${ }^{21,28-34,36-38,40-43}$ or having spinal chord injury ${ }^{23,25,35}$ with symptoms of spasticity, ${ }^{21-26,28-30,32,33,36-40,43}$ and/or pain $21,27,29,31,34,35,38$ and to abstain from any type of cannabinoids for 7 days prior to the entry and during the study. ${ }^{21,22,32,35,40,44}$ Exclusion criteria were most commonly having a disease of clinical importance, ${ }^{21,24,26,28-44}$ psychiatric disorders, ${ }^{21-26,31-41}$ being pregnant or lactating, ${ }^{23-25,32,35,36,40-43}$ or having hypersensitivity to cannabinoids, ${ }^{23,25,31-36,39,40}$.

Table 2 describes study characteristics related to intervention, control group, and assessed outcomes. All of the RCTs compared a cannabinoid substance to placebo. Nine of them used an oromucosal spray that delivers 2,7 milligrams of THC and 2,5 milligrams of CBD on each pump 21,24,29,31-35,37-40,44 four of them used synthetic

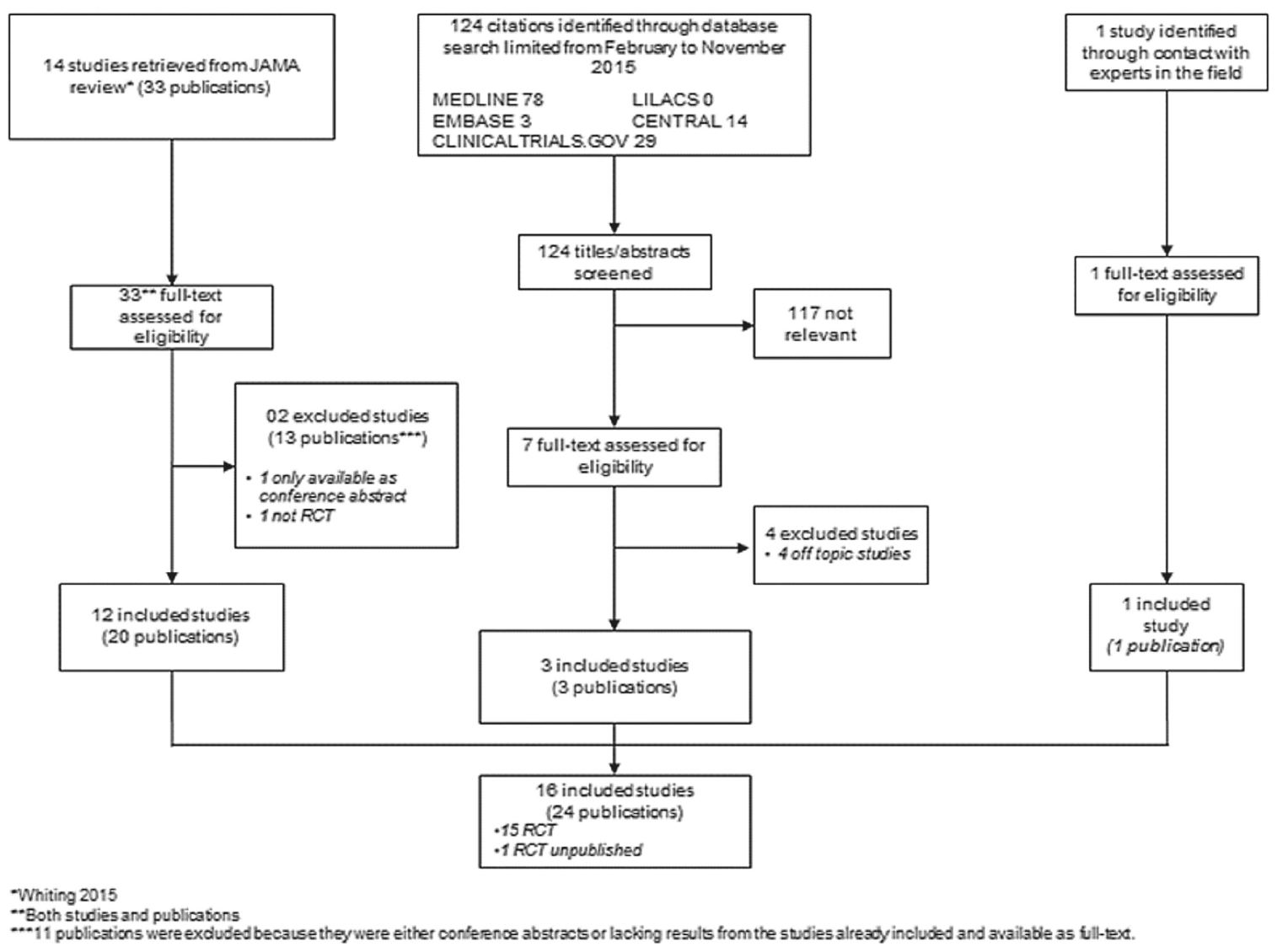

Fig. 1. Flow diagram of search results. 


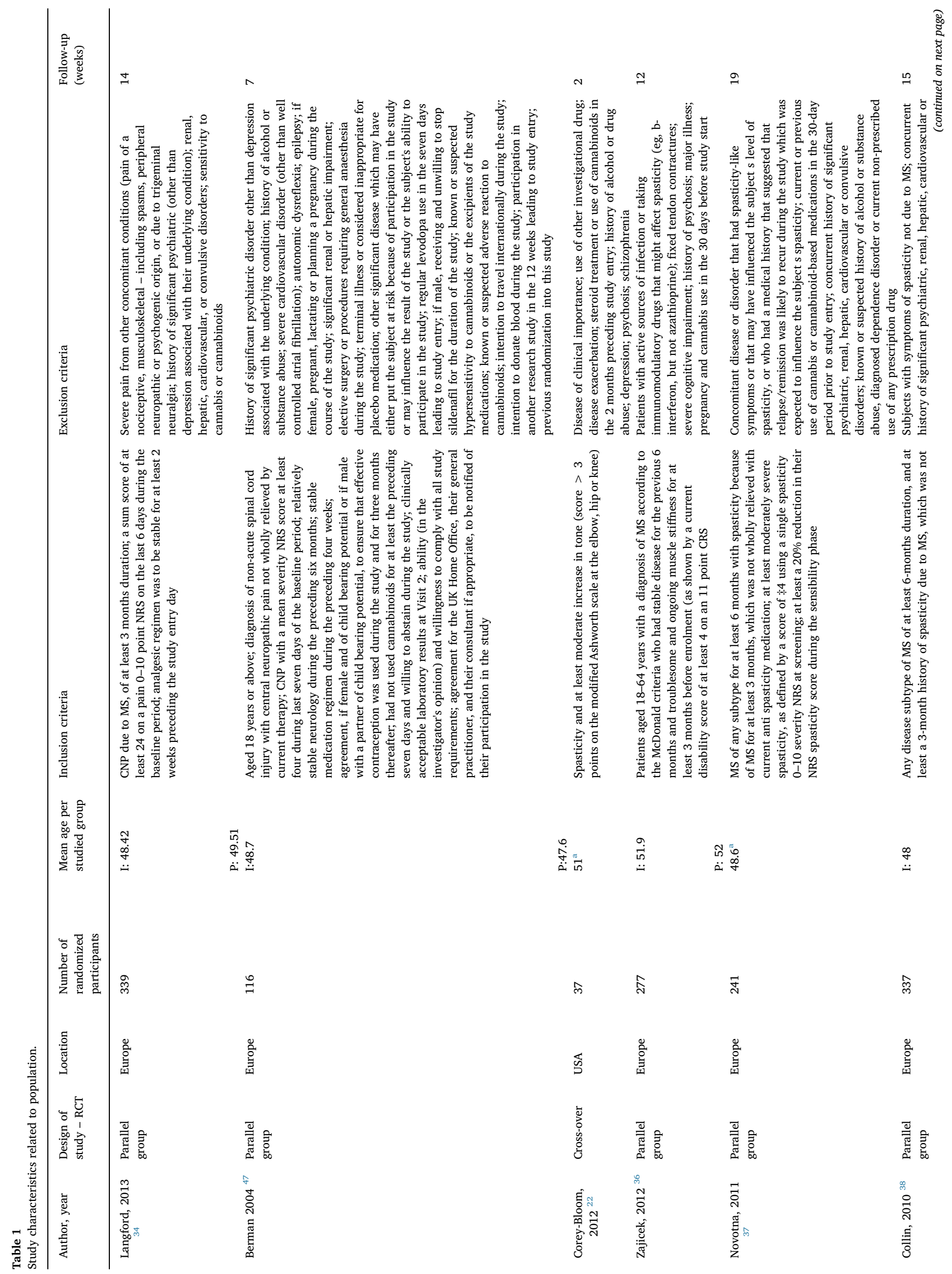




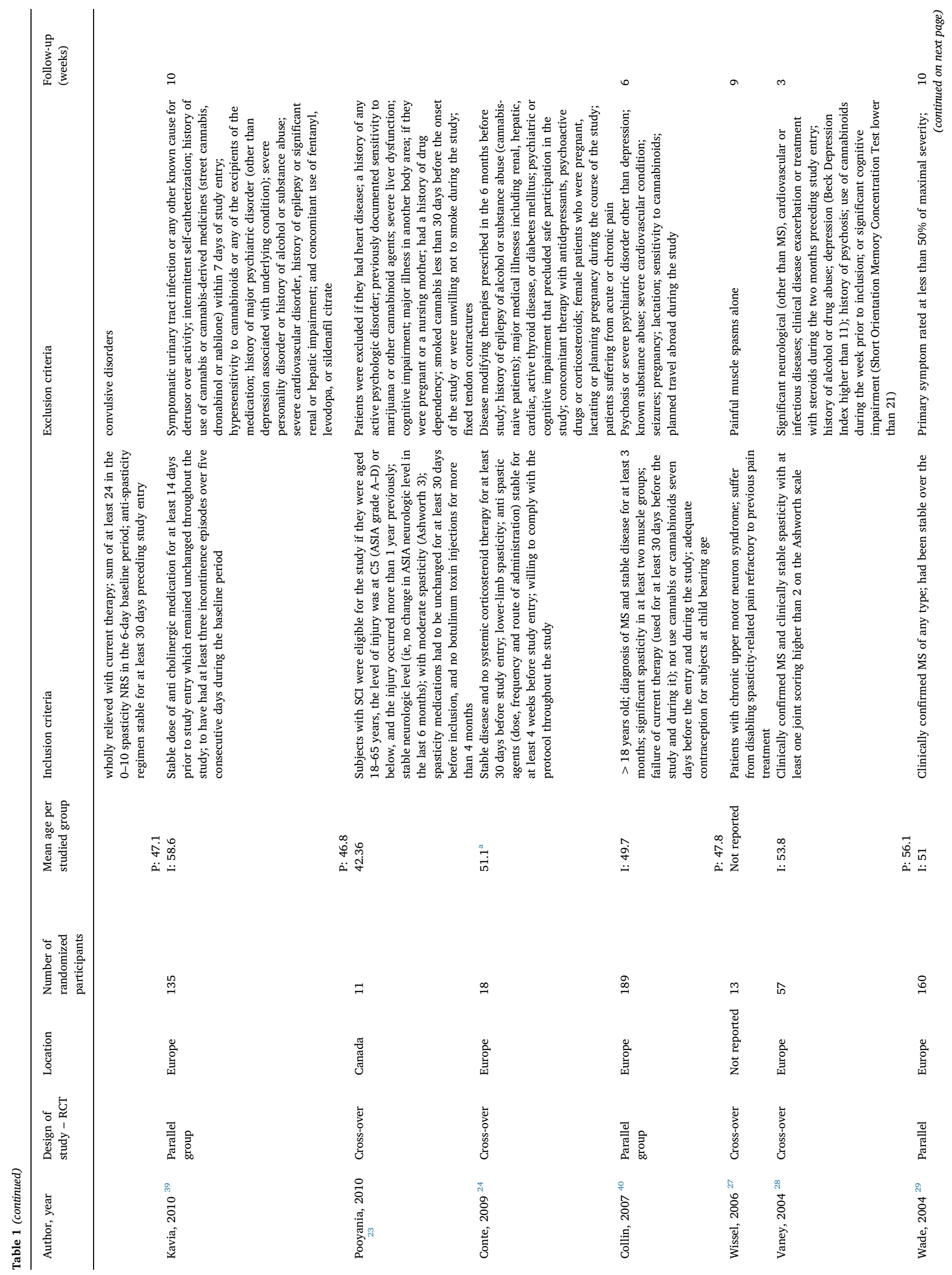




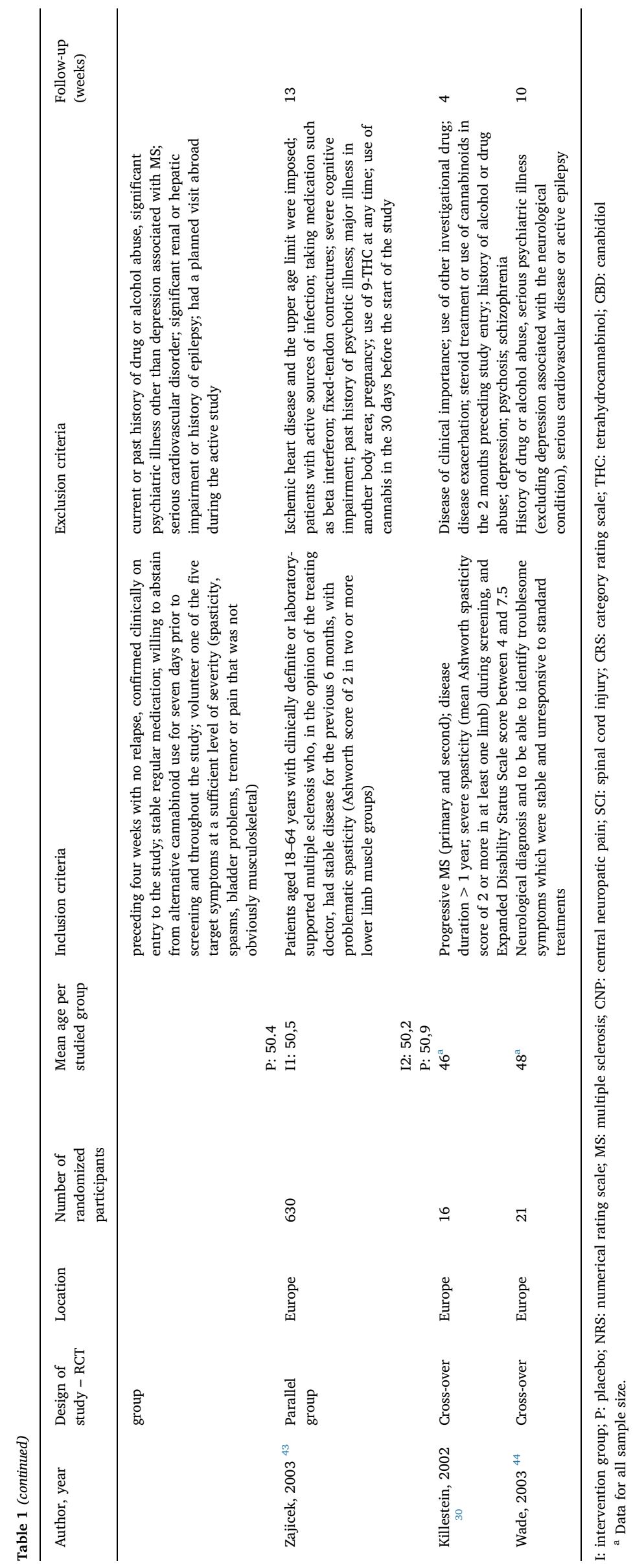




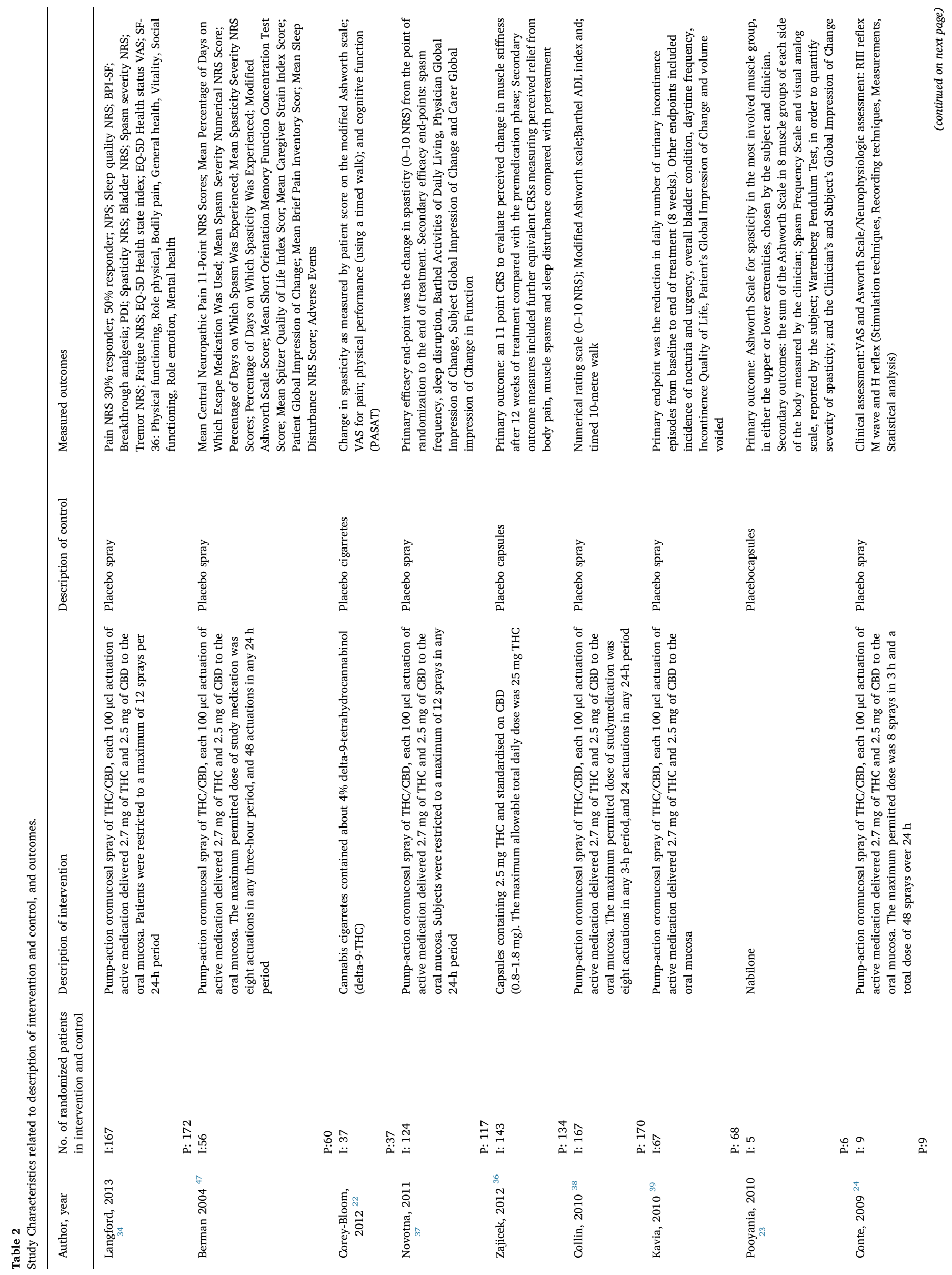




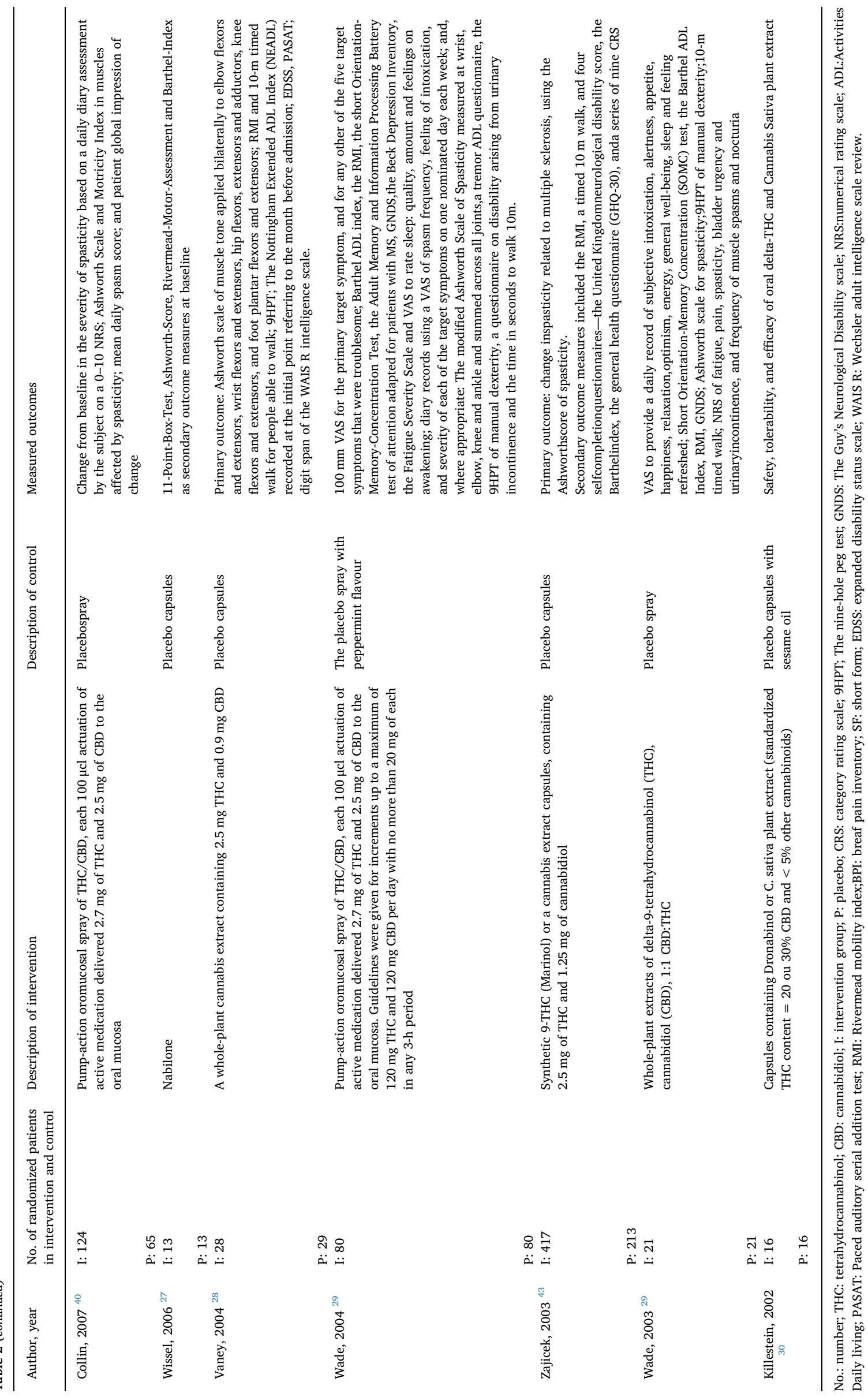




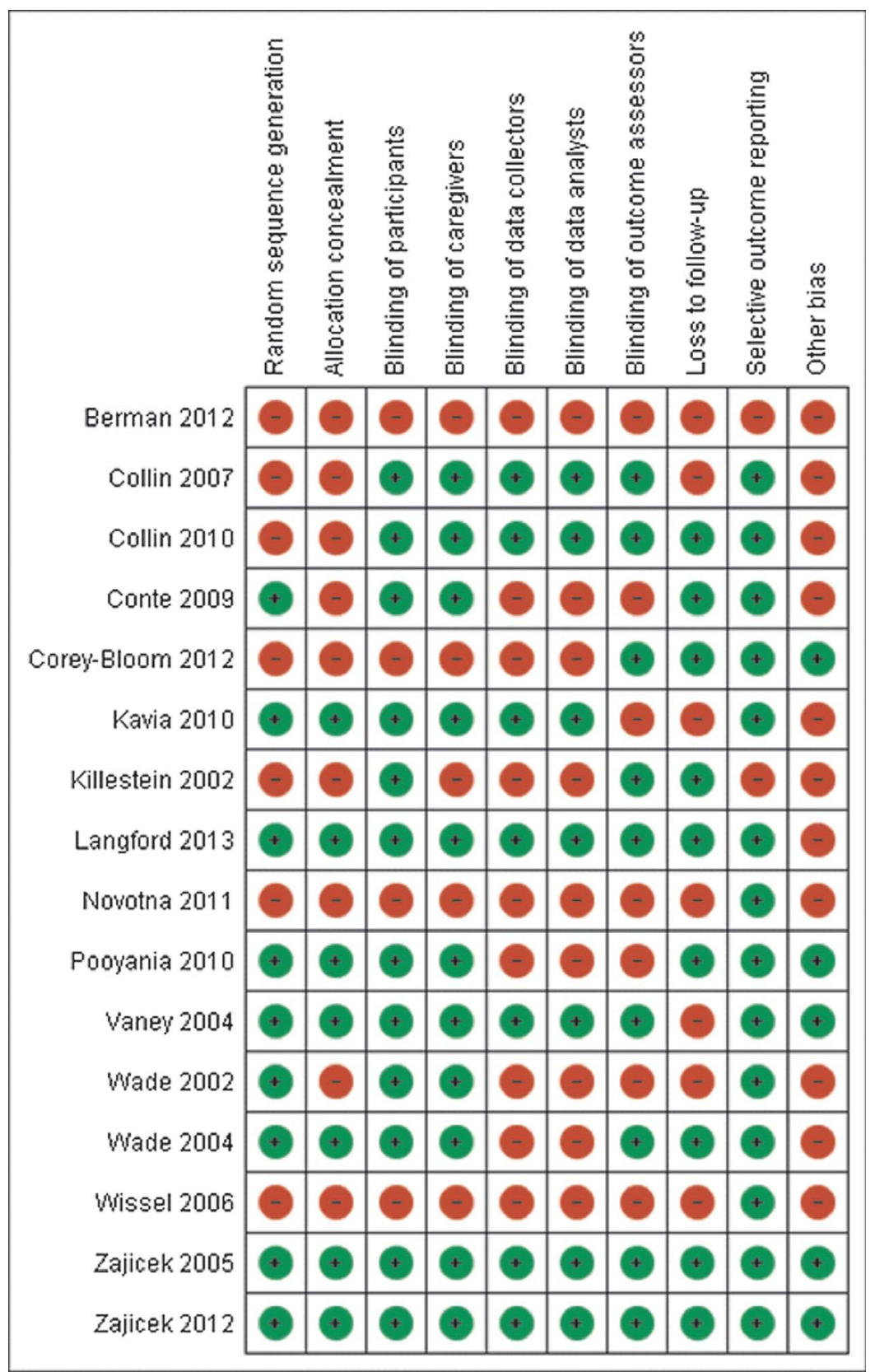

Fig. 2. Risk of bias assessment.

cannabinoids in capsules (one used dronabinol, ${ }^{30}$ one used marinol, ${ }^{41-43}$ two used nabilone, ${ }^{23,25,27}$ ), three used cannabis extracts in capsules ${ }^{22,28,30}$ and one used cannabis cigarettes. ${ }^{22,26}$ The most commonly described outcomes were spasticity, ${ }^{22-29,31-38,42-44}$ pain, ${ }^{22,26,31,34-36}$ functionality, ${ }^{22,26-29,31,34,35,41-44}$ and urinary symptoms, ${ }^{29,31,34,39,44}$.

\subsection{Risk of bias assessment}

Fig. 2 describes the risk of bias assessment for the RCTs. The major issue in the bias assessment was the risk of bias related to conflict of interest in the majority of the RCTs (68.7\%). Others were problems with random sequence $(43.75 \%)$, concealment of randomization $(56.25 \%)$, blinding of data collectors $(56,2 \%)$, data analysts $(56.2 \%)$, outcome assessors (43.75\%), the extent of missing outcome data (43.75\%). The CAMS study ${ }^{41-43}$ was the only one that was rated as low risk of bias for all assessed domains (Fig. 2).

\subsection{Outcomes}

\subsubsection{Spasticity}

Results from seven RCTs ${ }^{21-23,25,26,28,29,32,35,40,44}$ found a not statistically significant different between cannabinoids and placebo related to spasticity (Std. Mean Difference (SMD) 0.36 [Confidential interval (CI) $95 \%-0.17$ to $\left.0.88 ; \mathrm{p}=0.18 ; \mathrm{I}^{2}=88 \%\right]$ ). Certainty in evidence was rated down to low because of inconsistency, indirectness, risk of bias, missing outcome data ${ }^{35}$ lack of blinding of participants, ${ }^{22,26,35}$ and outcome assessors ${ }^{23,25,35,44}$ and random sequence and allocation concealment, ${ }^{22,26,32,35,40,44}$ (Fig. 3, Table 3).

\subsubsection{Spasm frequency}

Results from six RCTs ${ }^{21,23,25,28,29,32,35,40,44}$ found no statistically significant difference between cannabinoids and placebo related to spasm frequency (SMD 0.04 [CI 95\% -0.15 to $0.22 ; \mathrm{p}=0.70$; $\left.\left.\mathrm{I}^{2}=2 \%\right]\right)$. Certainty in evidence was rated down to moderate because of indirectness, risk of bias, due to missing outcome data ${ }^{35}$ and lack of 


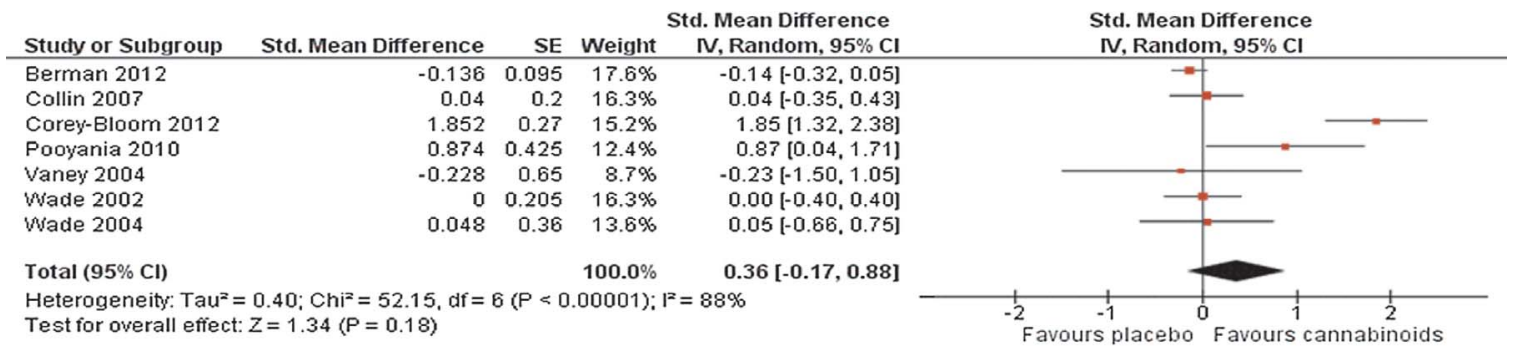

Fig. 3. Pooled analysis of spasticity comparing cannabinoids and placebo.

blinding of participants ${ }^{35}$ and outcome assessors ${ }^{23,25,35,44}$ and random sequence and allocation concealment, ${ }^{32,35,40,44}$ (Fig. 4, Table 3).

\subsubsection{Spasm severity}

Results from three RCTs ${ }^{23,25,35,44}$ found no statistically significant difference between cannabinoids and placebo related to spasm severity (SMD - 0,14 [CI 95\% - 0.63 to 0.36; $\left.\mathrm{p}=0.59 ; \mathrm{I}^{2}=0 \%\right]$ ). Certainty in evidence was rated down to moderate because of indirectness, risk of bias, missing outcome data ${ }^{35}$ lack of blinding of participants, ${ }^{35}$ and outcome assessors ${ }^{23,25,35,44}$ and random sequence and allocation concealment, ${ }^{26,44}$ (Fig. 5, Table 3).

\subsubsection{Pain}

Results from five RCTs $21,22,26,29,31,34,35,44$ found no statistically significant difference between cannabinoids and placebo related to pain (SMD - 0.02 [CI 95\% -0.39 to $0.35 ; \mathrm{p}=0.90 ; \mathrm{I}^{2}=0 \%$ ]). Certainty in evidence was rated down to moderate because of indirectness in all studies (Fig. 6, Table 3).

\subsubsection{Cognitive function}

Results from three RCTs ${ }^{22,26,28,44}$ found no statistically significant difference between cannabinoids and placebo related to cognitive function (SMD 0.55 [CI 95\% -3.33 to $\left.4.43 ; \mathrm{p}=0.78 ; \mathrm{I}^{2}=0 \%\right]$ ). Certainty in evidence was rated down to moderate because of indirectness, risk of bias, lack of blinding of participants ${ }^{22,26}$ and outcome assessors ${ }^{44}$ and random sequence and allocation concealment, ${ }^{22,26,44}$ (Fig. 7, Table 3).

\subsubsection{Daily activities}

Results from three RCTs ${ }^{21,29,44}$ found no statistically significant difference between cannabinoids and placebo related to daily activities (SMD 0.01 [CI 95\% -1.21 to $1.24 ; \mathrm{p}=0.98 ; \mathrm{I}^{2}=0 \%$ ]). Certainty in evidence was rated down to moderate because of indirectness in all studies (Fig. 8, Table 3).

\subsubsection{Motricity}

Results from four RCTs ${ }^{21,22,26,29,32,40}$ found no statistically significant difference between cannabinoids and placebo related to motricity (SMD 0.34 [CI $95 \%-0.60$ to $1.27 ; \mathrm{p}=0.48$; $\left.\mathrm{I}^{2}=0 \%\right]$ ). Certainty in evidence was rated down to moderate because of indirectness in all studies (Appendix Figure A2).

\subsubsection{Bladder function}

Results from one RCT ${ }^{21,29}$ found no statistically significant difference between cannabinoids and placebo related to bladder function (SMD - 0.06 [CI 95\% - 19.13 to $19.01 ; \mathrm{p}=0.99 ; \mathrm{I}^{2}$ not applicable]). Certainty in evidence was rated down to moderate because of indirectness in all studies (Appendix, Figure A3).

\subsection{Adverse events}

\subsubsection{Dizziness}

Results from 14 RCTs ${ }^{21,22,24,26,28-44}$ found a statistically significant difference favoring placebo over cannabinoids (RR 3.45 [CI 95\%
2.71-4.40; $\left.\left.\mathrm{p}<0.00001 ; \mathrm{I}^{2}=23 \%\right]\right)$. Certainty in evidence was rated down to moderate because of risk of bias, lack of random sequence ${ }^{30,37}$ and allocation concealment $22,24,26,30,37,44$ lack of blinding, ${ }^{21,29,37,39,44}$ missing outcome data, ${ }^{28,37}$ and, indirectness in all studies (Fig. 9, Table 4).

\subsubsection{Somnolence}

Results from 11 RCTs ${ }^{21,28,29-38,40,44}$ found a statistically significant difference favoring placebo over cannabinoids (RR 2.90 [CI 95\% 1.98-4.23; $\left.\mathrm{p}<0.00001 ; \mathrm{I}^{2}=0 \%\right]$ ). Certainty in evidence was rated down to moderate because of risk of bias, lack of random sequence $32,33,35,37,30,38$ and allocation concealment $24,27,30,32,33,40,41,44$ lack of blinding, ${ }^{21,24,29,30,35,37,44}$ missing outcome data ${ }^{28,37}$ selective outcome reporting, ${ }^{30}$ and, indirectness in all studies (Fig. 9, Table 4).

\subsubsection{Headache}

Results from 12 RCTs $21,22,24,26,28,29-32,34-37,39,40,44$ found no statistically significant difference comparing cannabinoids to placebo (RR 1.10 [CI 95\% 0.79-1.54; $\left.\mathrm{p}=0.57 ; \mathrm{I}^{2}=7 \%\right]$ ]). Certainty in evidence was rated down to moderate because of risk of bias, lack of random sequence $22,26,30,32,35,37,40$ and allocation concealment $21,22,24,26,30,32,37,40,44$ lack of blinding, ${ }^{21,24,26,29,30,35,37,39,44}$ missing outcome data, ${ }^{28,37,39,44}$ selective outcome reporting, ${ }^{30}$ and, indirectness in all studies (Fig. 9, Table 4).

\subsubsection{Nausea}

Results from 11 RCTs $21,22,24,26,28,29,31-40,44$ found a statistically significant difference favoring placebo over cannabinoids (RR 2.25 [CI 95\% $\left.\left.1.62-3.13 ; \mathrm{p}<0.00001 ; \mathrm{I}^{2}=0 \%\right]\right)$. Certainty in evidence was rated down to moderate because of risk of bias, lack of random sequence $22,26,32,33,35,37,38,40$ and allocation concealment $21,22,24,26,29,32,33,35,37,38,40$ lack of blinding, ${ }^{21,22,24,26,29,35,41,42,44}$ missing outcome data, ${ }^{37,39}$ and, indirectness in all studies (Fig. 9, Table 4).

\subsubsection{Dry mouth}

Results from 10 RCTs ${ }^{28,31-38,40-44}$ found a statistically significant difference favoring placebo over cannabinoids related to dry mouth (RR 2.82 [CI 95\% 2.06-3.85; $\mathrm{p}<0.00001 ; \mathrm{I}^{2}=0 \%$ ]). Certainty in evidence was rated down to moderate because of risk of bias, lack of random sequence $30,32,33,35,37,38,40$ and allocation concealment $30,32,33,35,37,38,40,44$ lack of blinding, ${ }^{30,35,37,44}$ missing outcome data, ${ }^{30,37}$ selective outcome reporting. ${ }^{30}$ and, indirectness in all studies (Fig. 9, Table 4).

More than 10 studies addressed adverse events; visual inspection of the funnel plot did not suggest publication bias (Appendix Figure A4).

\subsubsection{The cannabinoids in multiple sclerosis (CAMS) study}

The CAMS study ${ }^{41-43}$ was the largest study approaching cannabinoids versus placebo for spasticity; however there was no statistically significant difference regards improvement in spasticity between both studied groups (RR 1.47 [CI 95\% 0.99-1.28], 209 patients]). The study also reported the following non statistically-significant difference outcomes: spasm frequency (RR 1.29 [CI 95\% 0.92-1.80], 231 patients); daily activities (energy) (RR 1.02 [CI 95\% 0.69-1.51], 249 patients); and pain (RR 2.14 [CI 95\% 1.31to 3.49], 178 patients). 


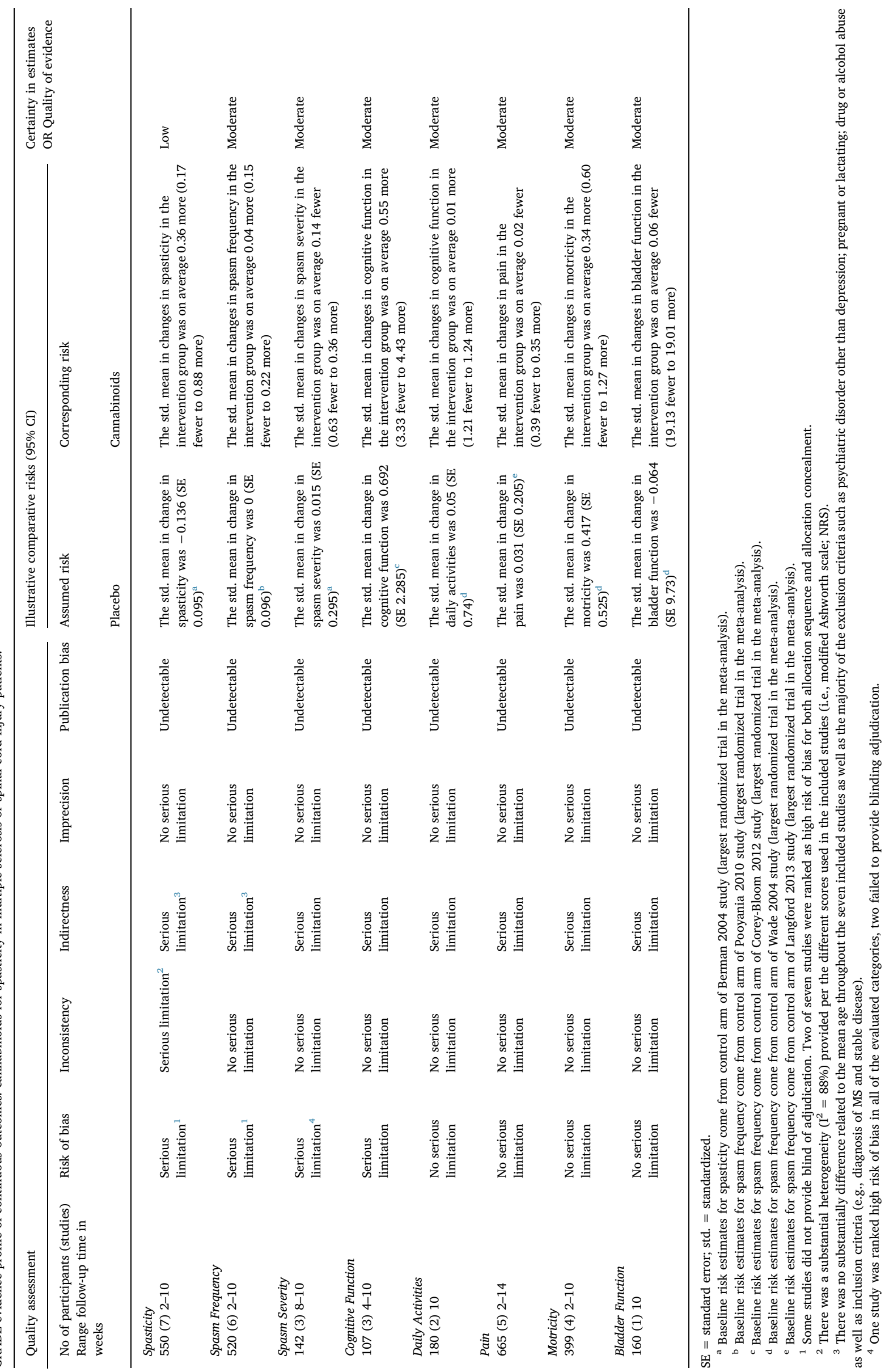




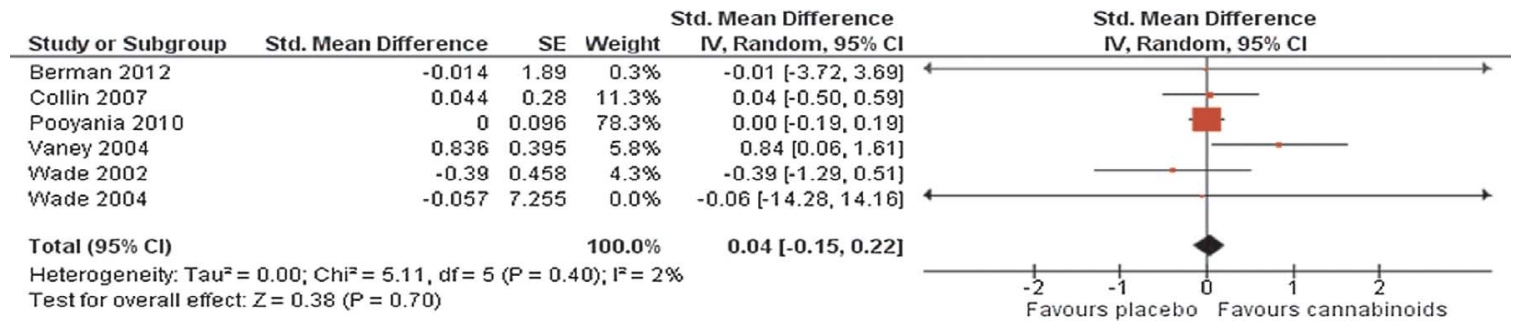

Fig. 4. Pooled analysis of spasm frequency comparing cannabinoids and placebo.

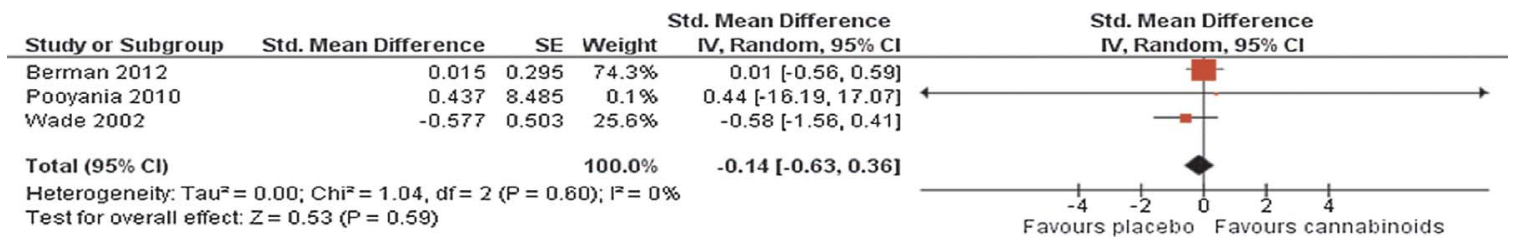

Fig. 5. Pooled analysis of spasm severity comparing cannabinoids and placebo.

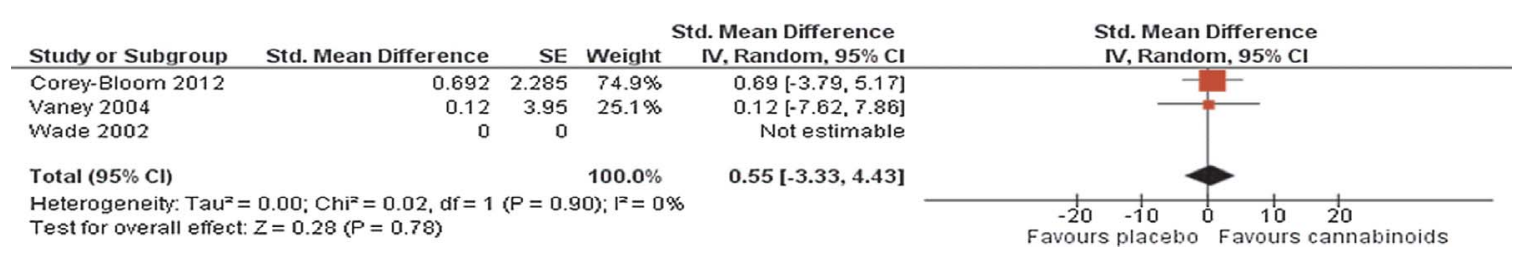

Fig. 6. Pooled analysis of pain comparing cannabinoids and placebo.

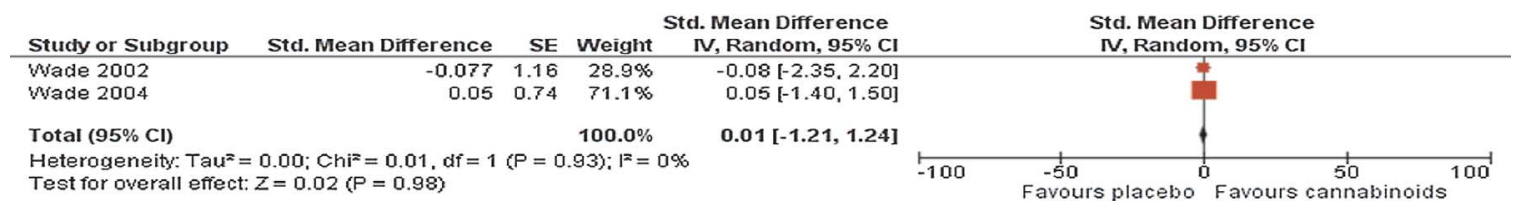

Fig. 7. Pooled analysis of cognitive function comparing cannabinoids and placebo.

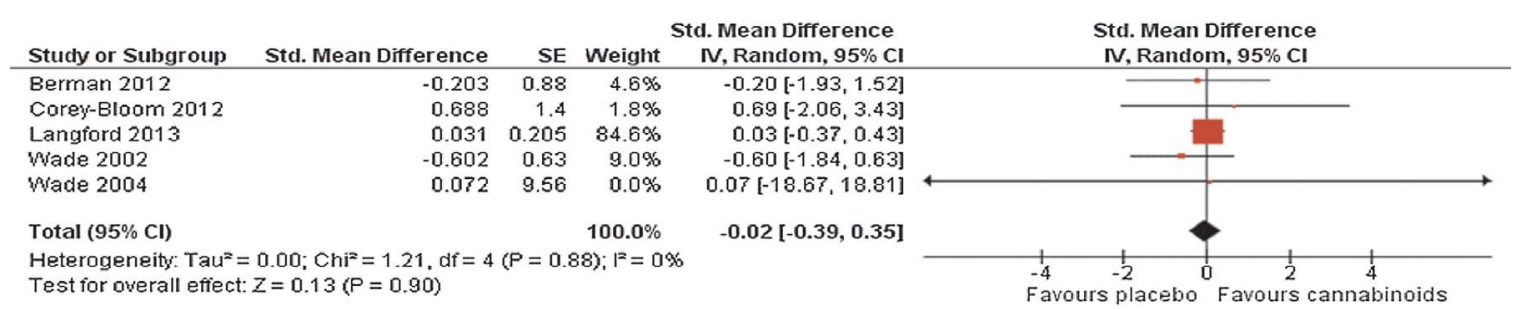

Fig. 8. Pooled analysis of daily activities comparing cannabinoids and placebo.

\section{Discussion}

\subsection{Main findings}

Based on the GRADE approach, meta-analysis of moderate certainty evidence from 16 eligible placebo-controlled trials including 2597 patients suggested a non-statistically significant improvement in spasm frequency and severity, cognitive function, daily activities, and motricity with cannabinoid use. Low certainty evidence showed a possible reduction in spasticity, although the association did not reach statistical significance either. Moderate certainty evidence also indicated a non-statistically significant deterioration in pain and bladder function.

Cannabinoid users experienced an approximately three-fold increased risk of dizziness, somnolence and dry mouth, and an approximately two-fold increased risk of nausea, relative to placebo. These adverse events are significantly more tolerable than those related to the use of the current spasticity therapy, such as respiratory depression, ataxia and hallucinations. A statistically significant difference favoring placebo over cannabinoids in relation to headaches was found as well.

\subsection{Strengths and limitations}

Strengths of this review include a broad search; evaluation of eligibility, risk of bias, and data abstraction independently and in duplicate; use of the GRADE approach in rating the quality of evidence; and focus on both absolute and relative effects of the intervention on patientimportant outcomes.

Potential limitations are related to the data available for this subject on the current literature. Trials often had outcomes reported incompletely, inadequate random sequence, and a fail of blinding due to the nature of the intervention, but for some studies also avoidable lack of blinding (outcome adjudication).

Another limitation of this review is the fact that most of the patients are using others concurrent active drugs such as interferon beta 1-b, glatiramer, and corticoids which can introduce bias in the true effects of 


\begin{tabular}{|c|c|c|c|c|c|c|}
\hline \multirow[b]{2}{*}{ Squdy or Subgroup } & \multicolumn{2}{|c|}{ Cannabinoids } & \multicolumn{2}{|c|}{ Placebo } & \multirow{2}{*}{ Weiglint } & \multirow{2}{*}{$\begin{array}{l}\text { Risk Ratio } \\
\text { M.H. Random. } 95 \% \mathrm{Cl}\end{array}$} \\
\hline & Events & rotal & Events & Totol & & \\
\hline \multicolumn{7}{|l|}{1.2 .1 Dizziness } \\
\hline Berman 2012 & 14 & 56 & 5 & 60 & $5.5 \%$ & $3.00[1.16,7.79]$ \\
\hline Collin 2007 & 40 & 128 & 7 & 65 & $8.2 \%$ & $3.00[1.42,6.31]$ \\
\hline Collin 2010 & 53 & 167 & 17 & 170 & $14.0 \%$ & $3.17\{1.92,5.25]$ \\
\hline Conte 2009 & 8 & 9 & 0 & 9 & $0.8 \%$ & $17.00|1.13,256.56|$ \\
\hline Corer Btoom 2012 & 8 & 30 & $i$ & 30 & $1.4 \%$ & $8.00[1.07,60.09]$ \\
\hline Karia 2010 & 12 & 67 & 4 & 88 & $4.4 \%$ & $3.04(1,03,8.97)$ \\
\hline Killestein 2002 & 6 & 16 & 3 & 16 & $3.7 \%$ & $2.00[0.60 .6 .64]$ \\
\hline Langford 2013 & 34 & 167 & 7 & 172 & $7.5 \%$ & $5.00[2.28,10.97]$ \\
\hline Novotaa 2011 & 4 & 124 & 0 & 117 & $0.7 \%$ & $3.50|0.45,156.10|$ \\
\hline vaney 2004 & 26 & 80 & 10 & 80 & $9.8 \%$ & $2.60[1.34,5.03]$ \\
\hline Wade 2002 & 0 & 21 & 1 & 21 & $0.6 \%$ & $0.33(0.01,7.74)$ \\
\hline Wade 2004 & 26 & 80 & 10 & 80 & $3.8 \%$ & $2.60[1.34,5.03\}$ \\
\hline Zajicek 2005 & 226 & 435 & 38 & 222 & $22.6 \%$ & $3.06[2.24,4.11]$ \\
\hline Zajicek 2012 & 89 & 143 & 10 & 134 & $10.3 \%$ & $8.34(4.53,15.34)$ \\
\hline Stibtotal $(95 \% \mathrm{Cl})$ & & 1519 & & 1244 & $100.0 \%$ & 3.45 (2.71, 4.40$)$ \\
\hline Tolat enents & 546 & & 113 & & & \\
\hline Heterogeneity Tata & 04,6 & $10, \cdots 0$ & -13 & & & \\
\hline
\end{tabular}

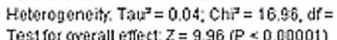

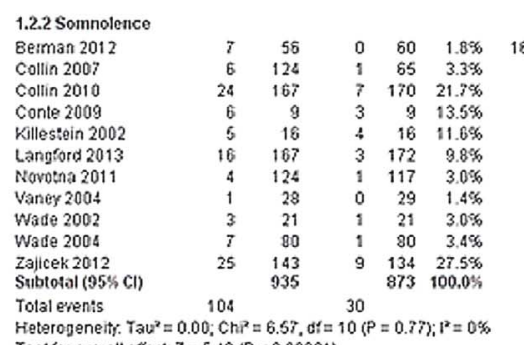

$16.05|0.94,274.71|$ $.15[0.39,25.57]$ $3.49[1.55,7.88\}$ $2.00[0.71,5.62]$
$1.25[0.41,3.82]$ $2500.41,3.821$
$5.4911 .03,88.511$ $5.49(1.63,18.51)$ 3.77 $(0.43,33.28]$ $3.10(0,13,73.12]$ $3.00(0.34,26.55]$ $2.60[1.26,5.37]$ $2.60[1.26,5.37]$

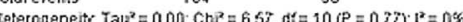
Tesifor operall effect $Z=5.49(P<0.00001)$

1.2.3 Headaclee

$80 \operatorname{mon} 2012$

Collin 2007

conte 2009

Corer-Bloom 2012

Karia 2010

Killestein 2002

Langford 2013

Norotna 2011

Vaney 2004

Wade 2002

Wato 2004

Zajicek 2012

Subtotal (95:

colal events 78

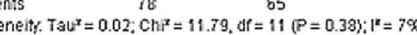

Tesifor operall effect: $Z=0.56(P=0.57)$

1.2. 4 Hausea

German 2012

Collin 2007

Collin 2010

Conte 2009

Corer-Bloom 2012

Kăna 2010

Langrord 2013

Novotna 2011

vaney 2004

Wade 2002

Wade 2004

Stibtotal $(95 \%$

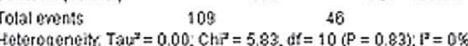

Testror overall effect $Z=4,81$ (P< 0,00001$)$

1.2.5 Dry Mouth

Berman 2012

Berman 201
Collin 2007

Collin 2007

Killestein 2002

Langford 2013

Navotaa 2011

Variog 2004

Wade 2002

Zajicek 2005

Zajicek 2012

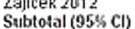

Tolal erents

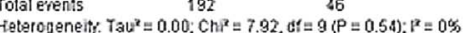

Test for orerall eflect: $Z=6.50$ ( $P<0.00001)$

$80 \quad 1,456 \quad 11,77|0,67,208,13|$

3. $6.386 \quad 1.05(0.33,3.351$

$30 \quad 11.1 \% \quad 1.17[0.44,3.05]$

$\begin{array}{lll}58 & 4.0 \% & 2.03(0.38,10.71)\end{array}$

$122 \quad 9280 \quad 1.20 .0 .41 .3501$

$117 \quad 4.2 \%$

$291.18 \%$

$1.20[0.41,3.50\}$ $0.38[0.07,1.91]$ $0.34[0.01,8.12]$ $2.00[0.41,9.77)$ $0.54[0,23,1.28)$
$1.03(0.59,1.80]$ $1.10[0.79,1.5 .9]$

$20 \quad 134 \quad 28.28$

$1.10[0.79,1.5 .41$

Risk Ratio

M.H. Random. $95 \% \mathrm{Cl}$

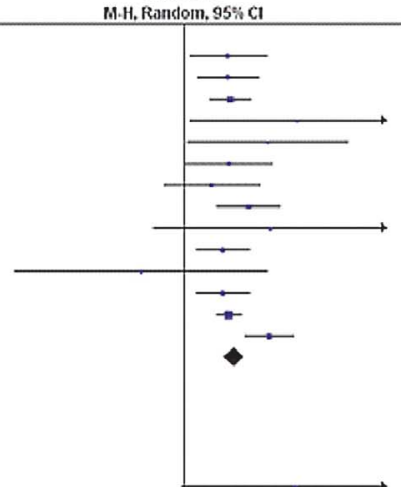

Fig. 9. Pooled analysis of adverse events comparing cannabinoids and placebo.

Tes1 for subntoup differences: $C h i^{2}=30.85 . d f=4(P<0.00001) . p^{z}=87.0 \%$

$2.14(0.56,8.16)$

$1.18[0.38,3.68]$

$3.17[1,92,5.25]$
$2.0010 .22,18.33]$

$2.0010 .22,18.331$

$4.00[0.47,33.73]$

$1.52[0.26,8.82]$
$1.91[0.78,4.68]$

$1.91(0.78,4.68)$

$2.36[0.47,11.92]$

$4.14,0.49,34.821$

$1.40(0.46,4.23)$

$2.25[1.62,3.13]$

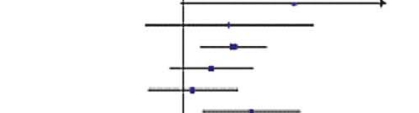

.
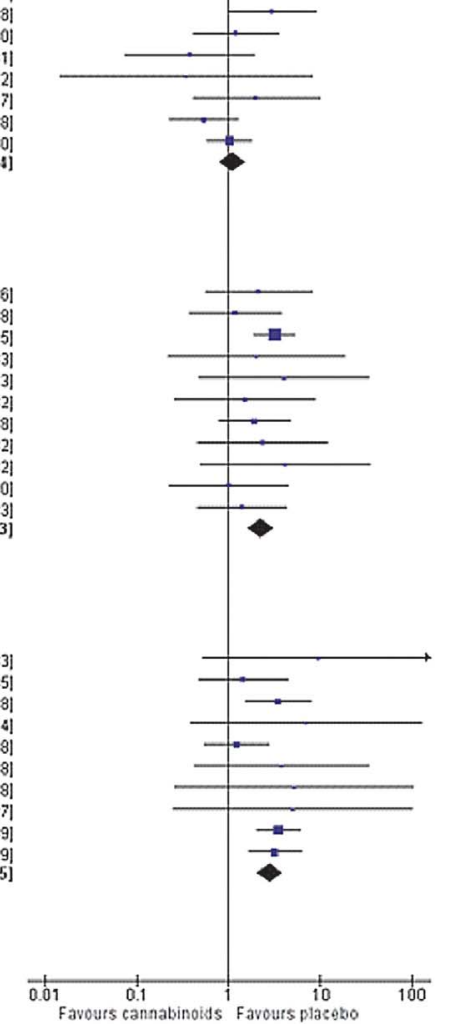

cannabinoids. The results of trials purporting beneficial effects of a new intervention could not ignore the effects of concurrent treatments.

Although this review presents some limitations, the issue is whether one should dismiss these results entirely or consider them bearing in mind the limitations. The latter represent our view of the matter.

\subsection{Relation to prior work}

A recent systematic review by Whiting and colleagues ${ }^{8}$ reviewed the literature from inception to April 2015, finding 79 studies with 6462 participants. The review reported moderate certainty, non-statistically significant improvements in spasticity due to multiple sclerosis, and chronic neuropathic or cancer pain with cannabinoid use ${ }^{8}$ Another review covering literature from 1948 to March 2015 via MEDLINE ${ }^{9}$ supported these findings, with six trials ( $\mathrm{n}=325$ patients) for chronic pain, six trials ( $\mathrm{n}=396$ patients) for neuropathic pain, and 12 trials ( $\mathrm{n}=1600$ patients) for multiple sclerosis-related spasticity supporting the potential efficacy of marijuana and cannabinoid 


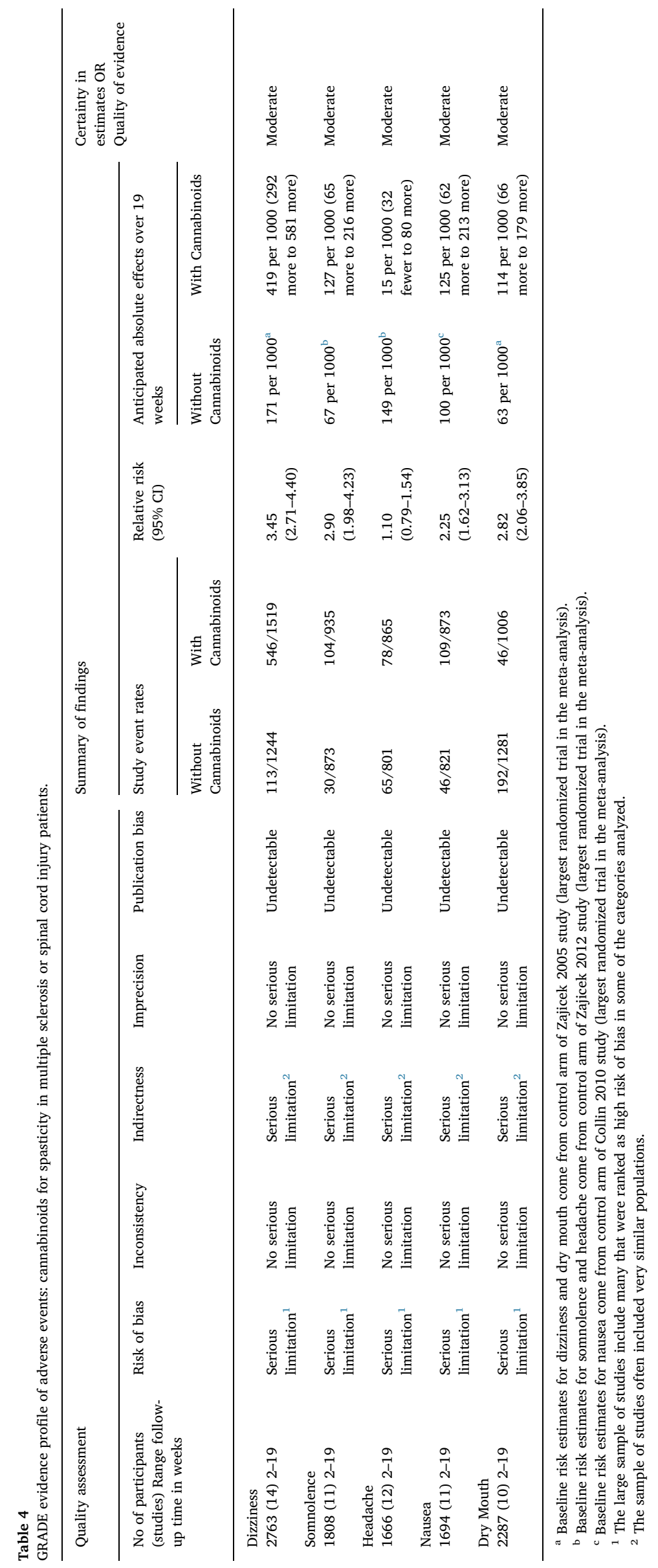


treatment for these indications.

In 2016 two articles sought to shed light on the understanding of the management of spasticity in MS patients. One of them ${ }^{45}$ is the largest multicentre observational study to date evaluating THC-CBD oromucosal spray effectiveness and tolerability in daily clinical practice, carried out in a routine outpatient setting. The other is a narrative review ${ }^{46}$ that describes findings from both randomized controlled phase III trials and recent studies on everyday clinical practice. Intending to expand and complement the comprehension on the subject, we engaged on a systematic and meta-analytic approach on this present review. Worthy of note is that both articles showed conclusions in line with our own findings, considering that spasticity is tremendously impactful on the patients and that it is currently being poorly managed. The reasons behind the inadequate approach of this ailment range from the lack of convincing evidence regarding the effectiveness and efficacy of the currently available therapy and it's AEs (which are more severe and frequent than cannabis based medicine showed to be), to the questionable reliability of the most widely used scale (Ashworth Scale) used to assess spasticity from the healthcare professional perspective. The studies also reinforced the importance of patient reported data such as VAS and NRS. Finally, THC-CBD oromucosal spray was interpreted as a safe and useful option for the management of spasticity resistant to available medication in MS patients.

Our update systematic review with 16 additional RCTs involving 2597 participants identified moderate-certainty evidence supporting a similar non-statistically significant improvement in spasm frequency and severity, and low certainty evidence supporting a reduction in spasticity. However, our results contrasted previous findings regarding cannabinoid effects on pain, where moderate-certainty evidence suggested a non-significant deterioration in multiple sclerosis or paraplegia.

\subsection{Implications for clinical practice and for research}

There is moderate certainty evidence regarding the impact of cannabinoids in spasticity (average 0.36 more spasticity; 0.17 fewer to 0.88 more) due to multiple sclerosis or paraplegia, and in adverse events such as dizziness (419 more dizziness/1000 over 19 weeks), somnolence (127 more somnolence/1000 over 19 weeks), and nausea (125 more somnolence/1000 over 19 weeks) and, dry mouth (114 more somnolence/1000 over 19 weeks). Further large-scale RCTs exploring the effectiveness of cannabinoids for spasticity and chronic pain in this patient population, as well as for other indications, are warranted.

\section{Funding and conflicts of interest}

Regina El Dib received a Brazilian Research Council National Counsel of Technological and Scientific Development (CNPq) scholarship (\#310953/2015-4).

\section{Acknowledgement}

We would like to thank José Eduardo Corrente from the Biostatistics Department, Biosciences Institute, São Paulo State University (Unesp), São Paulo, Brazil for his help with the statistical analysis.

\section{Appendix A. Supplementary data}

Supplementary data associated with this article can be found, in the online version, at http://dx.doi.org/10.1016/j.ctim.2017.08.010.

\section{References}

1 Goldstein J, Phadke CP, Ismail F, Boulias C. Spasticity health literacy among canadian family phisicians. Can J Neurol Sci. 2015;42:450-453.

2 Malfitano AM, Proto MC, Bifulco M. Cannabinoids in the management of spasticity associated with multiple sclerosis. Neuropsychiatr Dis Treat 2008:4:847-853.

3 Adams MM, Hicks AL. Spasticity after spinal cord injury. Spinal Cord. 2005;43:577-586

4 Sköld C, Levi R, Seiger A. Spasticity after traumatic spinal cord injury: nature, severity, and location. Arch Phys Med Rehabil. 1999;80:1548-1557.

5 Teive HAG, Zonta M, Kumagai Y. Treatment of spasticity: an update. Arq NeuroPsiquiatr. 1998;56:852-858.

6 Rocha FC, Dos Santos Junior JG, Stefano SC, da Silveira DX. Systematic review of the literature on clinical and experimental trials on the antitumor effects of cannabinoids in gliomas. J Neurooncol. 2014;116:11-24.

7 David Potter, The propagation, characterisation and optimisation of cannabis sativa L as a phytopharmaceutical. JP MlBiol CBiol FLS CMIOSH.

8 Whiting PF, Wolff RF, Deshpande S, et al. Cannabinoids for medical use: a systematic review and meta-analysis. JAMA. 2015;313:2456-2473.

9 Hill KP. Medical marijuana for treatment of chronic pain and other medical and psychiatric problems: a clinical review. JAMA. 2015;313:2474-2483.

10 Higgins JPT, Green S, eds. Cochrane Handbook for Systematic Reviews of Interventions Version 5.1.0 [updated March 2011]. The Cochrane Collaboration; 2011. Available from www.cochrane-handbook.org.

11 Moher D, Liberati A, Tetzlaff J, Altman DG, PRISMA Group. Preferred reporting items for systematic reviews and meta-analyses: the PRISMA statement. BMJ. 2009;339:b2535.

12 Moher D, Cook DJ, Eastwood S, Olkin I, Rennie D, Stroup DF. Improving the quality of reports of meta-analyses of randomised controlled trials: the QUOROM statement: quality of reporting of meta-analyses. Lancet. 1999;354:1896-1900.

13 Guyatt GH, Busse JW. Modification of Cochrane Tool to Assess Risk of Bias in Randomized Trials. 2017; 2017http://distillercer.com/resources/.

14 Guyatt GH, Oxman AD, Vist GE, et al. GRADE: an emerging consensus on rating quality of evidence and strength of recommendations. BMJ. 2008;336:924-926.

15 Guyatt GH, Oxman AD, Vist G, et al. GRADE guidelines: 4. Rating the quality of evidence-study limitations (risk of bias). J Clin Epidemiol. 2011;64:407-415.

16 Guyatt GH, Oxman AD, Kunz R, et al. GRADE guidelines 6. Rating the quality of evidence-imprecision. J Clin Epidemiol. 2011;64:1283-1293.

17 Guyatt GH, Oxman AD, Kunz R, et al. GRADE working group. GRADE guidelines: 7. Rating the quality of evidence-inconsistency. J Clin Epidemiol. 2011:64:1294-1302.

18 Guyatt GH, Oxman AD, Kunz R, et al. GRADE guidelines: 8. Rating the quality of evidence-indirectness. J Clin Epidemiol. 2011;64:1303-1310.

19 Guyatt GH, Oxman AD, Montori V, et al. GRADE guidelines: 5. Rating the quality of evidence-publication bias. J Clin Epidemiol. 2011;64:1277-1282.

20 The Nordic Cochrane Centre. The Cochrane Collaboration. Review Manager (RevMan) 5.3. Copenhagen: The Nordic Cochrane Centre, The Cochrane Collaboration; 2011

21 An Investigation of Delta-9-Tetrahydrocannabinol (THC) and Cannabidiol (CBD) in Multiple Sclerosis Patients. 2017; 2017http://ClinicalTrials.gov/show/NCT01610700.

22 Corey-Bloom J, Wolfson T, Gamst A, et al. Smoked cannabis for spasticity in multiple sclerosis: a randomized, placebo-controlled trial. CMAJ. 2012;184:1143-1150.

23 Pooyania S, Ethans K, Szturm T, Casey A, Perry D. A randomized, double-blinded, crossover pilot study assessing the effect of nabilone on spasticity in persons with spinal cord injury. Arch Phys Med Rehabil. 2010;91:703-707.

24 Conte A, Bettolo CM, Onesti E, et al. Cannabinoid-induced effects on the nociceptive system: a neurophysiological study in patients with secondary progressive multiple sclerosis. Eur J Pain. 2009;13:472-477.

25 Randomized Double Blind Cross over Study for Nabilone in Spasticity in Spinal Cord Injury Persons. 2017; 2017http://ClinicalTrials.gov/show/NCT00623376.

26 Short-Term Effects of Medicinal Cannabis Therapy on Spasticity in Multiple Sclerosis. 2017; 2017http://ClinicalTrials.gov/show/NCT00248378.

27 Wissel J, Haydn T, Müller J, et al. Low dose treatment with the synthetic cannabinoid Nabilone significantly reduces spasticity-related pain: a double-blind placebo-controlled cross-over trial. J Neurol. 2006;253:1337-1341.

28 Vaney C, Heinzel-Gutenbrunner M, Jobin P, et al. Efficacy, safety and tolerability of an orally administered cannabis extract in the treatment of spasticity in patients with multiple sclerosis: a randomized, double-blind, placebo-controlled, crossover study. Mult Scler. 2004;10:417-424.

29 Wade DT, Makela P, Robson P, House H, Bateman C. Do cannabis-based medicinal extracts have general or specific effects on symptoms in multiple sclerosis? A doubleblind, randomized, placebo-controlled study on 160 patients. Mult Scler. 2004;10:434-441

30 Killestein J, Hoogervorst EL, Reif M, et al. Safety, tolerability, and efficacy of orally administered cannabinoids in MS. Neurology. 2002;58:1404-1407.

31 Sativex Versus Placebo when Added to Existing Treatment for Central Neuropathic Pain in M.S. 2017; 2017http://ClinicalTrials gov/show/NCT00391079.

32 A Study of Sativex for Relief of Spasticity in Subjects with Multiple Sclerosis. 2017; 2017http://ClinicalTrials.gov/show/NCT00711646.

33 A Study to Evaluate the Efficacy of Sativex in Relieving Symptoms of Spasticity Due to Multiple Sclerosis. 2017; 2017http://ClinicalTrials.gov/show/NCT01599234.

34 Langford RM, Mares J, Novotna A, et al. A double-blind, randomized, placebo-controlled, parallel-group study of THC/CBD oromucosal spray in combination with the existing treatment regimen, in the relief of central neuropathic pain in patients with multiple sclerosis. J Neurol. 2013;260:984-997.

35 A Study of Cannabis Based Medicine Extracts and Placebo in Patients with Pain Due to Spinal Cord Injury. 2017; 2017http://ClinicalTrials.gov/show/NCT01606202.

36 Zajicek JP, Hobart JC, Slade A, Barnes D, Mattison PG, MUSEC Research Group. Multiple sclerosis and extract of cannabis: results of the MUSEC trial. $J$ Neurol Neurosurg Psychiatry. 2012;83:1125-1132.

37 Novotna A, Mares J, Ratcliffe S, et al. A randomized, double-blind, placebo-controlled, parallel-group, enriched-design study of nabiximols* (Sativex $\left(^{\circ}\right)$ ), as add-on therapy, in subjects with refractory spasticity caused by multiple sclerosis. Eur $J$ 
Neurol. 2011:18:1122-1131.

38 Collin C, Ehler E, Waberzinek G, et al. A double-blind, randomized, placebo-controlled, parallel-group study of Sativex, in subjects with symptoms of spasticity due to multiple sclerosis. Neurol Res. 2010;32:451-459.

39 Kavia RB, De Ridder D, Constantinescu CS, Stott CG, Fowler CJ. Randomized controlled trial of Sativex to treat detrusor overactivity in multiple sclerosis. Mult Scler. 2010;16:1349-1359.

40 Collin C, Davies P, Mutiboko IK, Ratcliffe S, Sativex Spasticity in MS Study Group. Randomized controlled trial of cannabis-based medicine in spasticity caused by multiple sclerosis. Eur J Neurol. 2007;14:290-296.

41 Freeman RM, Adekanmi O, Waterfield MR, Waterfield AE, Wright D, Zajicek J. The effect of cannabis on urge incontinence in patients with multiple sclerosis: a multicentre, randomised placebo-controlled trial (CAMS-LUTS). Int Urogynecol J Pelvic Floor Dysfunct 2006:17:636-641.

42 Zajicek JP, Sanders HP, Wright DE, et al. Cannabinoids in multiple sclerosis (CAMS) study: safety and efficacy data for 12 months follow up. J Neurol Neurosurg Psychiatry.
2005:76:1664-1669.

43 Zajicek J, Fox P, Sanders H, et al. Cannabinoids for treatment of spasticity and other symptoms related to multiple sclerosis (CAMS study): multicentre randomised placebo-controlled trial. Lancet. 2003;8(362):1517-1526.

44 Wade DT, Robson P, House H, Makela P, Aram J. A preliminary controlled study to determine whether whole-plant cannabis extracts can improve intractable neurogenic symptoms. Clin Rehabil. 2003;17:21-29.

45 Patti F, Messina S, Solaro C, et al. Efficacy and safety of cannabinoid oromucosal spray for multiple sclerosis spasticity. J Neurol Neurosurg Psychiatry. 2016;87:944-951.

46 Zettl UK, Rommer P, Hipp P, Patejdl R. Evidence for the efficacy and effectiveness of THC-CBD oromucosal spray in symptom management of patients with spasticity due to multiple sclerosis. Ther Adv Neurol Disord. 2016;9(1):9-30.

47 Berman JS, Symonds C, Birch R. Efficacy of two cannabis based medicinal extracts for relief of central neuropathic pain from brachial plexus avulsion: results of a randomised controlled trial. Pain. 2004;112(3):299-306. 